\title{
Isohedral Polyomino Tiling of the Plane*
}

\author{
K. Keating and A. Vince \\ Department of Mathematics, University of Florida, \\ Gainesville, FL 32611, USA \\ \{keating,vince\}@math.ufl.edu
}

\begin{abstract}
A polynomial time algorithm is given for deciding, for a given polyomino $P$, whether there exists an isohedral tiling of the Euclidean plane by isometric copies of $P$. The decidability question for general tilings by copies of a single polyomino, or even periodic tilings by copies of a single polyomino, remains open.
\end{abstract}

\section{Introduction}

Besides its place among topics in recreational mathematics, polyomino tiling of the plane is relevant to decidability questions in logic and data storage questions in parallel processing. A polyomino is a rookwise connected tile formed by joining unit squares at their edges. We always assume that our polyominoes are made up of unit squares in the Cartesian plane whose centers are lattice points and whose sides are parallel to the coordinate axes. A polyomino consisting of $n$ unit squares is referred to as an $n$-omino. Polyominoes were introduced in 1953 by Golomb; his book Polyominoes, originally published in 1965 [3], has recently been revised and reissued [5]. See also his survey article [6] for an overview of polyomino tiling problems.

One of the first questions to arise in the subject was the following: Given a single polyomino $P$, can isometric copies of $P$ tile the plane? It is known that every $n$-omino for $n \leq 6$ tiles the plane. Of the 108 7-ominoes, only 4 of them, shown in Fig. 1, do not tile the plane [8, pp. 501-502]. The following is a long-standing open problem.

Question 1.1. Does there exists an algorithm to determine whether a given polyomino $P$ tiles the plane?

\footnotetext{
* The first author was partially supported by NSF Grant 9500982.
} 

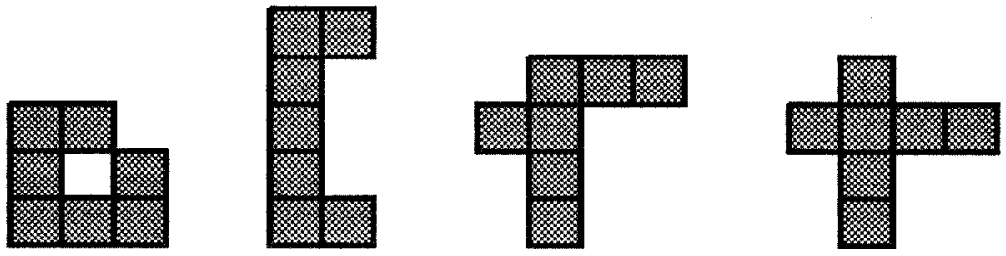

Fig. 1. Heptominoes which do not tile the plane.

Golomb [7] has shown that the problem of determining whether an arbitrary finite set of polyominoes tiles the plane (assuming unlimited copies of each) is equivalent to the corresponding problem for Wang tiles, a problem known to be undecidable [13]. On the other hand, there does exist an algorithm for determining whether a single polyomino tiles the plane by translation [14, Theorem 6.1].

A tiling of the plane by copies of an $n$-omino $P$ will be referred to as a $P$-tiling. For a tiling $\mathcal{T}$, define the symmetry group of $\mathcal{T}$ to be the group $G(\mathcal{T})$ of isometries of the plane that preserve $\mathcal{T}$. A $P$-tiling $\mathcal{T}$ is called isohedral if $G(\mathcal{T})$ acts transitively on the tiles in $\mathcal{T}$. A tiling $\mathcal{T}$ is periodic if $G(\mathcal{T})$ contains translations in two linearly independent directions; otherwise $\mathcal{T}$ is nonperiodic. In the case of a periodic tiling, the group of translations that preserves the tiling is called the translation group. If the translation group itself acts transitively on the tiles in $\mathcal{T}$, then $\mathcal{T}$ is called a lattice tiling.

A lattice tiling is necessarily isohedral and an isohedral tiling is necessarily periodic. However, as we shall see below, there are polyominoes that admit an isohedral tiling but no lattice tiling and polyominoes that admit a periodic tiling but no isohedral tiling. A polyomino $P$ (not a tiling!) is called aperiodic if there exists a $P$-tiling, but no $P$-tiling is periodic. Figure 2(a) is a portion of a nonperiodic tiling by a 3-omino, but this 3 -omino is not aperiodic since it also has the periodic tiling shown in Fig. 2(b).

Question 1.2. Does there exist an aperiodic polyomino?

In fact, it is an open question whether there exists a single aperiodic tile, polyomino or not. Penrose [11] has constructed aperiodic sets of tiles, some of which have as few as two elements. For example, there are uncountably many tilings of the plane by copies of Penrose "kites" and "darts", but no such tiling is periodic. In addition, Penrose has produced an aperiodic set consisting of three polyominoes [6, Figure 27].

An affirmative answer to Question 1.2 would make Question 1.1 especially delicate. On the other hand, a negative answer to Question 1.2 implies that Question 1.1 is equivalent to Question 1.3 below. Even so, to our knowledge, no algorithm is known to determine whether a given polyomino tiles the plane periodically.

Question 1.3. Does there exist an algorithm to determine whether a given polyomino $P$ tiles the plane periodically?

The following relevant question also appears to be open. 


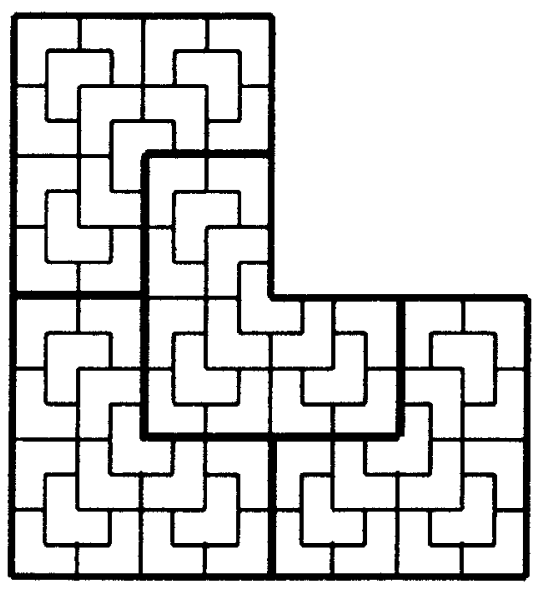

(a)

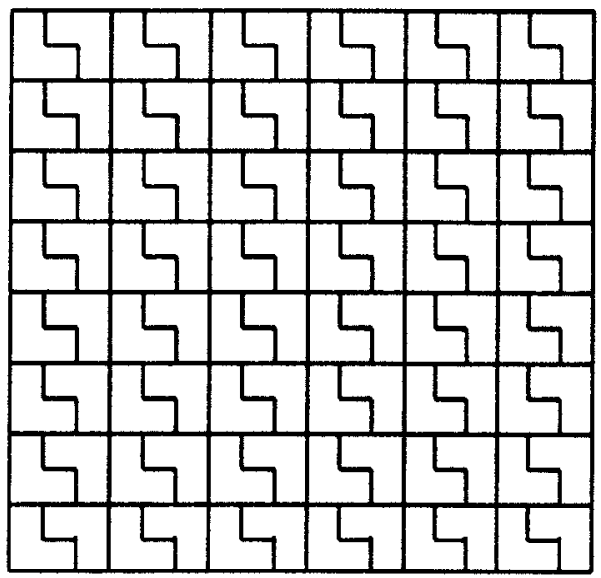

(b)

Fig. 2. Two tilings by a 3-omino.

Question 1.4. Does there exist an integer $\beta$, independent of $n$, such that, for every $n$-omino $P$ that tiles the plane periodically, there is a periodic tiling $\mathcal{T}$ by copies of $P$ for which $(\mathbb{Z} \times \mathbb{Z}: L) \leq \beta n$, where $L$ is the translation subgroup of $G(\mathcal{T})$ ?

Given such a $\beta$, one could devise an algorithm to decide whether a given polyomino has a periodic tiling. Concerning positive evidence for the existence of $\beta$, for all the small examples of $n$-ominoes $(n<10)$ that appear in the literature, if there is a tiling at all, then there is one with the property that $(\mathbb{Z} \times \mathbb{Z}: L)$ equals $n, 2 n, 4 n$, or $8 n$. On the negative side, there exist periodic tilings for which $(\mathbb{Z} \times \mathbb{Z}: L) / n$ is large. For instance, in [2] there is an example of an asymmetric tiling of a $19 \times 28$ rectangle by a certain 7-omino $P$. By translating this tiling of the rectangle horizontally and vertically we get a periodic tiling of the plane whose symmetry group is a translation group $L$ with $(\mathbb{Z} \times \mathbb{Z}: L)=19 \cdot 28=76 n$. For this particular $P$, though, there is another periodic tiling with translation group $L^{\prime}$ such that $\left(\mathbb{Z} \times \mathbb{Z}: L^{\prime}\right)=14=2 n$. It appears that Question 1.4 may be difficult to answer.

Klarner [9] defines the order of a polyomino $P$ to be the smallest number of copies of $P$ which tile a rectangle. If there is no rectangle which is tiled by copies of $P$, then the order of $P$ is not defined. It is known that there exist polyominoes of all orders divisible by 4 [4], and of infinitely many orders congruent to 2 modulo 8 [10]. The following question remains open.

Question 1.5. Does there exist a nonrectangular polyomino of odd order?

In [12] it is shown that there are no polyominoes of order 3.

If the original problem is restricted to tilings by translations of a polyomino $P$ (no 


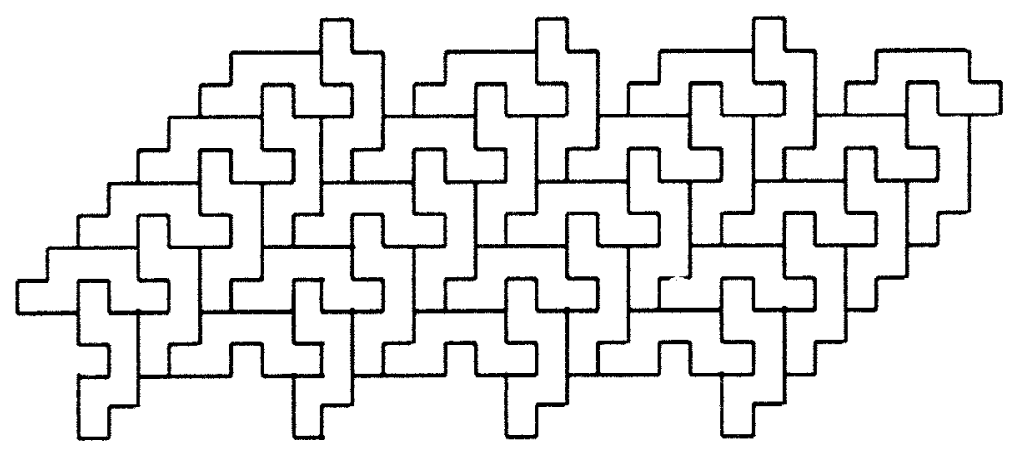

Fig. 3. A nonisohedral tiling.

rotations, reflections, or glide reflections allowed), then the subtleties mentioned above vanish. If there exists a tiling using only translations of $P$, then there also exists a lattice tiling by copies of $P$ [14, Theorem 5.4]. Several necessary and sufficient conditions for the existence of a lattice tiling by a polyomino are given in [1]. An efficient algorithm to determine whether a given polyomino admits a lattice tiling appears in Section 4 of this paper.

The main result of this paper is an answer to Question 1.1 restricted to isohedral tilings. In Section 4 we provide a polynomial time algorithm that determines whether a given polyomino $P$ admits an isohedral tiling of the plane. Note that there exist polyominoes, such as those in Figs. 4 and 5, which tile the plane isohedrally but admit no lattice tiling. On the other hand, there also exist polyominoes that tile the plane periodically but do not admit an isohedral tiling, for instance the 7-omino in Fig. 3.

The basic idea underlying the algorithm is as follows. If an isohedral $P$-tiling $\mathcal{T}$ exists, we let $L$ denote the translation subgroup of $G(\mathcal{T})$. There is a fundamental region $F \subset \mathbb{R}^{2}$ for the action of $L$ which is the union of a finite number of tiles in $\mathcal{T}$. In fact, we show in Section 3 that if there is an isohedral $P$-tiling at all, then there is one such that $F$ consists of one, two, or four copies of $P$. The tiling $\mathcal{T}$ consists of the set of translations of the copies of $P$ in $F$ by the translation group $L \leq \mathbb{Z}^{2}$; in order for the tiling to be isohedral $L$ must satisfy certain constraints which depend on $F$. The algorithm has two stages. First, it constructs configurations of copies of $P$ to form $F$; second, for each such $F$ it attempts to construct an appropriate translation subgroup $L$.

We have implemented the algorithm using the MATLAB package. We found that for every $n \leq 6$, every $n$-omino $P$ admits an isohedral tiling. In fact, for each such $P$ there is an isohedral tiling such that the fundamental region for the translation subgroup $L$ consists of either a single copy of $P$ or two copies of $P$ which are mapped to each other by a $180^{\circ}$ rotation. The 7 -ominoes in Fig. 1 admit no tiling, hence obviously admit no isohedral tiling. Figure 3 shows a nonisohedral tiling of the plane by a certain 7-omino $P_{1}$. In fact, $P_{1}$ admits both isohedral and nonisohedral tilings. Figure 4(a) is a diagram from [8, p. 506] of a tiling by a certain 7-omino $P_{2}$, a tiling that is claimed to be isohedral. In fact, this tiling is not isohedral, but there does exists an isohedral $P_{2}$-tiling shown in Fig. 4(b). Notice that the fundamental region for this tiling consists of four copies of $P_{2}$ : $P_{2}$ itself, the image $P_{2}^{\prime}$ of $P_{2}$ under a glide reflection through an axis of slope 1 , and the 


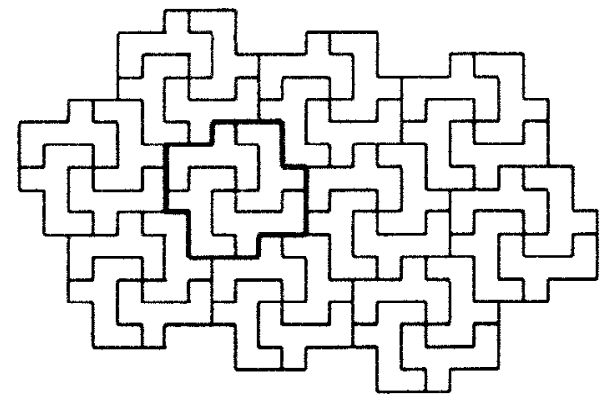

(a)

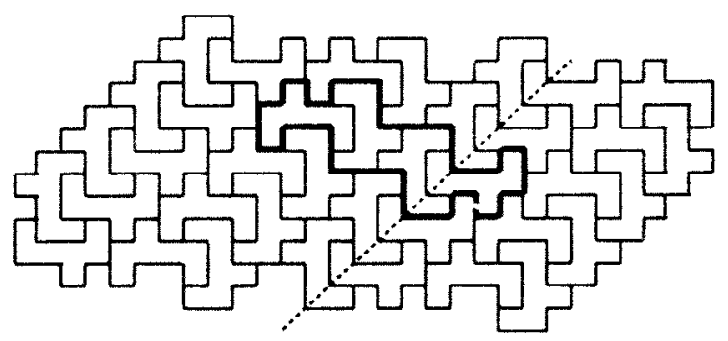

(b)

Fig. 4. Tilings by a 7-omino: Nonisohedral (a) and isohedral (b) tiling.

images of $P_{2}$ and $P_{2}^{\prime}$ under a $180^{\circ}$ rotation. Any fundamental region for an isohedral tiling by $P_{2}$ must consists of four such copies. Figure 5 shows a tiling by a certain 8 -omino $P_{3}$ where the fundamental region consists of four copies of $P_{3}: P_{3}$ itself and the images of $P_{3}$ under $90^{\circ}, 180^{\circ}$, and $270^{\circ}$ rotations. For this 8 -omino a fundamental region for an isohedral tiling must consists of four such copies. For $n \leq 7$ there is no $n$-omino which requires these four rotations to construct an isohedral tiling.

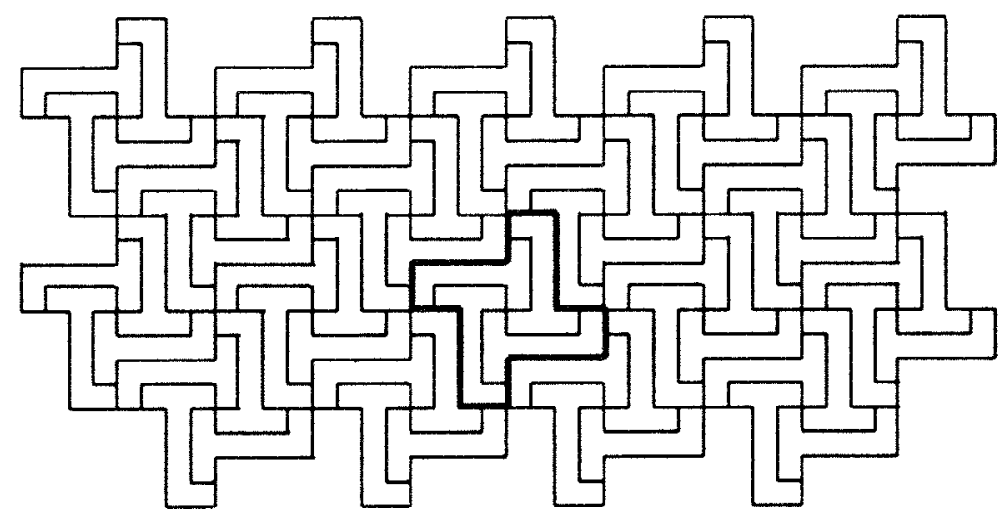

Fig. 5. An isohedral tiling by an 8-omino. 


\section{The Symmetry Group of a Polyomino Tiling}

We begin by describing the group $\Gamma$ of isometries of the plane that preserve the standard lattice $\mathbb{Z} \times \mathbb{Z}$. Any isometry of the plane can be written uniquely in the form $g(\mathbf{x})=$ $\varphi(\mathbf{x})+\mathbf{a}$, where $\varphi$ is an element of the orthogonal group $\mathbb{O}_{2}(\mathbb{R})$ and $\mathbf{a} \in \mathbb{R}^{2}$ is a translation vector. Since $g$ preserves $\mathbb{Z}^{2}$ we must have $\varphi \in D_{8}$ and $\mathbf{a} \in \mathbb{Z}^{2}$, where the dihedral group $D_{8}$ is the group of isometries of the unit square centered at $(0,0)$. This leads to a homomorphism $\Psi: \Gamma \rightarrow D_{8}$ defined by $\Psi(g)=\varphi$ and an exact sequence

$$
1 \longrightarrow \mathbb{Z}^{2} \longrightarrow \Gamma \longrightarrow D_{8} \longrightarrow 1
$$

There are three basic categories of lattice-preserving isometries of the plane, namely translations by an element of $\mathbb{Z}^{2}$, rotations through an angle of $90^{\circ}, 180^{\circ}$, or $270^{\circ}$, and glide reflections. A glide reflection is the composition of a reflection with a translation in the direction of the axis of the reflection. In this terminology a reflection is the special case of a glide reflection with trivial translation. If $\sigma \in \Gamma$ is a glide reflection, the axis of $\sigma$ must be parallel to one of the coordinate axes, or have slope \pm 1 . The geometric type of an element of $\Gamma$ (translation, rotation through a particular angle, or glide reflection through an axis with a particular slope) is determined by its image in $D_{8}$.

The group $\Gamma$ can be described in terms of generators and relations as follows. Let $x$ denote translation by one unit to the right and let $y$ denote translation by one unit upward. Let $r$ denote the $90^{\circ}$ counterclockwise rotation about $(0,0)$, and let $f$ denote the reflection through the line of slope 1 through the origin. Then $x, y, r, f$ generate $\Gamma$ and satisfy the following relations:

$$
\begin{aligned}
& r^{4}=f^{2}=1, \quad y x y^{-1}=x, \quad r x r^{-1}=y, \quad r y r^{-1}=x^{-1}, \\
& f x f^{-1}=y, \quad f y f^{-1}=x, \quad f r f^{-1}=r^{3} .
\end{aligned}
$$

It follows that $\Gamma \cong \mathbb{Z}^{2} \rtimes D_{8}$ is a semidirect product of $\mathbb{Z}^{2}$ and $D_{8}$, and that every element $g \in \Gamma$ is written uniquely in the form $g=x^{i} y^{j} r^{k} f^{l}$ with $0 \leq k \leq 3$ and $0 \leq l \leq 1$. In terms of these generators $\Psi: \Gamma \rightarrow D_{8}$ is given by the formula $\Psi\left(x^{i} y^{j} r^{k} f^{l}\right)=r^{k} f^{l}$.

To help us prove the next proposition we list the ten subgroups of $D_{8}$ :

Order 8: $\quad D_{8}=\left\langle r, f: r^{4}=f^{2}=1, f r f^{-1}=r^{3}\right\rangle$.

Order 4: $\quad\left\langle r^{2}, f\right\rangle \cong Z_{2} \times Z_{2},\left\langle r^{2}, r f\right\rangle \cong Z_{2} \times Z_{2},\langle r\rangle \cong Z_{4}$.

Order 2: $\quad\langle f\rangle \cong Z_{2},\langle r f\rangle \cong Z_{2},\left\langle r^{2} f\right\rangle \cong Z_{2},\left\langle r^{3} f\right\rangle \cong Z_{2},\left\langle r^{2}\right\rangle \cong Z_{2}$.

Order 1: $\{1\}$.

We say that two such subgroups are geometrically equivalent if there is an automorphism of $D_{8}$ which maps one to the other. There are six geometric equivalence classes of subgroups of $D_{8}$; in fact the first two subgroups of order 4 are geometrically equivalent (generated by a reflection and a $180^{\circ}$ rotation), and the first four subgroups of order 2 are geometrically equivalent (generated by a reflection). 
Proposition 2.1. Let $\mathcal{T}$ be an isohedral $P$-tiling, and let $G \leq \Gamma$ be a group that acts transitively on the tiles in $\mathcal{T}$. Then there is a subgroup $H$ of $G$ that acts simply transitively on the tiles in $\mathcal{T}$.

Proof. Let $P_{0}$ be a tile in $\mathcal{T}$ and let $K \leq G$ be the stabilizer of $P_{0}$. Since $G$ acts transitively on the tiles in $\mathcal{T}$, there is a one-to-one correspondence between the left cosets of $K$ in $G$ and the tiles in $\mathcal{T}$, given by $g K \leftrightarrow g\left(P_{0}\right)$. Therefore it suffices to find a subgroup $H$ of $G$ whose elements are a complete set of coset representatives for $G / K$. Equivalently, we need $H K=G$ and $H \cap K=\{1\}$.

Let $\psi: G \rightarrow D_{8}$ denote the restriction to $G$ of $\Psi: \Gamma \rightarrow D_{8}$. Set $\bar{G}=\psi(G)$ and $\bar{K}=\psi(K)$. Also let $L=\operatorname{ker}(\psi)$ denote the translation subgroup of $G$. Suppose there is $\bar{H} \leq \bar{G}$ such that $\bar{H} \bar{K}=\bar{G}$ and $\bar{H} \cap \bar{K}=\{1\}$; then define $H=\psi^{-1}(\bar{H}) \leq G$. Since $\operatorname{ker}(\psi)=L \leq H$ and $\psi(H K)=\psi(G)$ we have $H K=G$. In addition, since $L \cap K=\{1\}$ we have $H \cap K=\{1\}$. Therefore $H=\psi^{-1}(\bar{H})$ acts simply transitively on the tiles in $\mathcal{T}$.

We now consider the question of when there exists $\bar{H} \leq \bar{G}$ which satisfies the conditions of the preceding paragraph. For any $\bar{G} \leq D_{8}$, if $\bar{K}=\bar{G}$, then $\bar{H}=\{1\}$ satisfies the conditions, while if $\bar{K}=\{1\}$, then $\bar{H}=\bar{G}$ works. If $\bar{G} \cong Z_{2} \times Z_{2}$, then for any $\bar{K} \leq \bar{G}$ we easily find $\bar{H} \leq \bar{G}$ satisfying the conditions. Suppose now that $\bar{G}=D_{8}$. Then if $\bar{K} \cong Z_{2}$ is generated by a reflection $r^{i} f$ we let $\bar{H}=\langle r\rangle$; if $\bar{K}=\langle r\rangle \cong Z_{4}$ we let $\bar{H}=\langle f\rangle$; and if $\bar{K} \cong Z_{2} \times Z_{2}$ we let $\bar{H}=\left\langle r^{i} f\right\rangle$, where $r^{i} f$ is any reflection not contained in $\bar{K}$.

It remains only to consider the two cases $\bar{G}=\langle r\rangle, \bar{K}=\left\langle r^{2}\right\rangle$ and $\bar{G}=D_{8}, \bar{K}=\left\langle r^{2}\right\rangle$. In these cases there is no $\bar{H} \leq \bar{G}$ such that $\bar{H} \bar{K}=\bar{G}$ and $\bar{H} \cap \bar{K}=\{1\}$, so we must use more delicate methods to construct $H$. Let $\rho \in G$ be a $90^{\circ}$ counterclockwise rotation, and let $\tau_{1}, \tau_{2}$ be the generators for the translation subgroup $L$ of $G$ supplied by Lemma 2.2 below. Let $H=\left\langle\tau_{1} \tau_{2}, \tau_{1} \tau_{2}^{-1}, \rho\right\rangle$; we claim that $H \cap K=\{1\}$. Since $\bar{K}=\left\langle r^{2}\right\rangle$ the only possibility for a nontrivial element in $H \cap K$ is a $180^{\circ}$ rotation, which we can write as $\eta_{0}=\tau_{1}^{a} \tau_{2}^{b} \rho^{2}$. Since $\eta_{0} \in H$ the integers $a$ and $b$ must have the same parity. This implies $\eta_{0}=\rho_{0}^{2}$ where $\rho_{0}=\tau_{1}^{(b+a) / 2} \tau_{2}^{(b-a) / 2} \rho \in G$ is a $90^{\circ}$ rotation. We then have $\eta_{0}\left(\rho_{0} P_{0}\right)=\rho_{0}\left(\eta_{0} P_{0}\right)=\rho_{0} P_{0}$, so by Lemma 2.3 below we get $\rho_{0} P_{0}=P_{0}$. This implies $\rho_{0} \in K$, which violates the assumption $\bar{K}=\left\langle r^{2}\right\rangle$. Therefore $H \cap K=\{1\}$. In the case $\bar{G}=\langle r\rangle, \bar{K}=\left\langle r^{2}\right\rangle$ we have $(G: H)=|K|=2$ and hence $H K=G$. Therefore in this case $H$ acts simply transitively on the tiles in $\mathcal{T}$.

We claim that the remaining case $\left(\bar{G}=D_{8}, \bar{K}=\left\langle r^{2}\right\rangle\right)$ does not actually occur. To prove this we first show that the subgroup $G_{0}=\langle L, \rho\rangle$ of $G$ acts transitively on the tiles in $\mathcal{T}$. Let $\eta_{0}=\tau_{1}^{a} \tau_{2}^{b} \rho^{2} \in K$ be a $180^{\circ}$ rotation which stabilizes $P_{0}$. The arguments above show that $\eta_{0} \notin H$, and hence that $a$ and $b$ have opposite parity. Let $P_{1}$ be another tile in $\mathcal{T}$; we need to show there is $\sigma \in G_{0}$ such that $\sigma P_{0}=P_{1}$. The stabilizer in $G$ of $P_{1}$ also contains a $180^{\circ}$ rotation $\eta_{1}=\tau_{1}^{c} \tau_{2}^{d} \rho^{2}$ such that $c$ and $d$ have opposite parity. For any $\lambda=\tau_{1}^{i} \tau_{2}^{j} \in L$ we have $\lambda \eta_{0} \lambda^{-1}=\tau_{1}^{2 i+a} \tau_{2}^{2 j+b} \rho^{2}$ and $(\lambda \rho) \eta_{0}(\lambda \rho)^{-1}=\tau_{1}^{2 i-b} \tau_{2}^{2 j+a} \rho^{2}$. Therefore there is $\sigma \in G_{0}=\langle L, \rho\rangle$ such that $\sigma \eta_{0} \sigma^{-1}=\eta_{1}$. It follows that $\eta_{1} \sigma P_{0}=\sigma \eta_{0} P_{0}=\sigma P_{0}$, so by Lemma 2.3 we have $\sigma P_{0}=P_{1}$. Now since $G_{0}$ acts transitively on the tiles in $\mathcal{T}$ we have $G_{0} K=G$. Since $G_{0}$ and $K$ contain no glide reflections, but $G$ does, this is a contradiction. 
Lemma 2.2. Let $G$ be an infinite subgroup of $\Gamma$ which contains a $90^{\circ}$ rotation $\rho$. Then the translation subgroup $L$ of $G$ is a square lattice generated by translations $\tau_{1}$ and $\tau_{2}$ such that $\rho \tau_{1} \rho^{-1}=\tau_{2}$ and $\rho \tau_{2} \rho^{-1}=\tau_{1}^{-1}$.

Proof. We may assume that $\rho=x^{k} y^{l} r$ for some $k, l \in \mathbb{Z}$. Let $\tau_{1}=x^{i} y^{j} \in L$ be a nonzero translation of minimum length. Set $\tau_{2}=\rho \tau_{1} \rho^{-1}=x^{-j} y^{i}$. Then $\tau_{2}$ is a translation with the same length as $\tau_{1}$ but in a perpendicular direction. The group $\left\langle\tau_{1}, \tau_{2}\right\rangle$ is a square lattice which is equal to $L$ by the minimality of the length of $\tau_{1}$. We have $\rho \tau_{1} \rho^{-1}=\tau_{2}$ by definition, and $\rho \tau_{2} \rho^{-1}=\rho^{2} \tau_{1} \rho^{-2}=\tau_{1}^{-1}$.

Lemma 2.3. Let $\eta_{0} \in G$ be a $180^{\circ}$ rotation. Then $\eta_{0}$ stabilizes at most one tile in $\mathcal{T}$.

Proof. It suffices to show that if $\eta_{0}$ stabilizes a tile $P_{0}$, then the center $O$ of $\eta_{0}$ is in the interior of $P_{0}$. Observe that $P_{0}$ must be simply connected since copies of $P_{0}$ tile the plane. Therefore if $O$ is not in the interior of $P_{0}$ there is a curve $\Pi$ from $O$ to infinity which is a homeomorphic image of $[0, \infty)$ and does not intersect $P_{0}$ except possibly at $O$. Then $\Pi \cup \eta_{0} \Pi$ is invariant under $\eta_{0}$ and also does not intersect $P_{0}$ except possibly at $O$. Therefore $\Pi \cup \eta_{0} \Pi$ separates the interior of $P_{0}$ into at least two components. This violates the assumption that $P_{0}$ is rookwise connected. Therefore $O$ is in the interior of $P_{0}$.

\section{Nonlattice Tilings}

Let $\mathcal{T}$ be an isohedral $P$-tiling and let $H \leq \Gamma$ be a group which acts simply transitively on the tiles in $\mathcal{T}$. Let $L \leq H$ be the translation subgroup of $H$, and let $h_{1}, \ldots, h_{k}$ be coset representatives for $H / L$. Then $h_{1}(P), \ldots, h_{k}(P)$ do not overlap, and $P^{\prime}=$ $h_{1}(P) \cup \cdots \cup h_{k}(P)$ tiles the plane by translation by $L$. In this section we consider this construction in the opposite direction. Given $h_{1}, \ldots, h_{k} \in \Gamma$ such that $h_{1}(P), \ldots, h_{k}(P)$ do not overlap, let $P^{\prime}=h_{1}(P) \cup \cdots \cup h_{k}(P)$. We want to find a $P^{\prime}$-tiling of the plane by translation by a lattice $L \leq \mathbb{Z}^{2}$ such that $H=L h_{1} \cup \cdots \cup L h_{k}$ is a group. Given such a $P^{\prime}$-tiling $\mathcal{T}^{\prime}$ we let $\mathcal{T}$ be the $P$-tiling induced by $\mathcal{T}^{\prime}$. Then $H$ acts simply transitively on the tiles in $\mathcal{T}$, and hence $\mathcal{T}$ is isohedral.

If $H \leq \Gamma$ has translation subgroup $L$, then $H / L$ is isomorphic to a subgroup of $D_{8} \cong \Gamma / \mathbb{Z}^{2}$. Therefore we may assume that $h_{1}, \ldots, h_{k}$ are coset representatives for the elements of some subgroup of $D_{8}$. We have five cases to consider, corresponding to the geometric equivalence classes of nontrivial subgroups of $D_{8}$.

Proposition 3.1. Let $\rho \in \Gamma$ be a $180^{\circ}$ rotation such that $P$ and $\rho(P)$ do not overlap, and set $P^{\prime}=P \cup \rho(P)$. Let $\mathcal{T}^{\prime}$ be a $P^{\prime}$-tiling of the plane by translations by a lattice $L \subset \mathbb{Z}^{2}$, and let $\mathcal{T}$ denote the $P$-tiling induced by $\mathcal{T}^{\prime}$. Then $H=\langle L, \rho\rangle$ acts simply transitively on the tiles in $\mathcal{T}$.

Proof. Since $L$ acts simply transitively on the tiles in $\mathcal{T}^{\prime}$ it suffices to show $H=L \cup L \rho$. If $\tau \in L$, then $\rho \tau \rho^{-1}=\tau^{-1}$, so $\rho$ normalizes $L$. Since $\rho$ has order 2 this implies $\langle L, \rho\rangle=L \cup L \rho$. 
Proposition 3.2. Let $\rho \in \Gamma$ be a $90^{\circ}$ rotation such that $P, \rho(P), \rho^{2}(P)$, and $\rho^{3}(P)$ do not overlap, and set $P^{\prime}=P \cup \rho(P) \cup \rho^{2}(P) \cup \rho^{3}(P)$. Let $\mathcal{T}^{\prime}$ be a $P^{\prime}$-tiling of the plane by translations by a lattice $L \subset \mathbb{Z}^{2}$, and let $\mathcal{T}$ denote the $P$-tiling induced by $\mathcal{T}^{\prime}$. Then $H=\langle L, \rho\rangle$ acts simply transitively on the tiles in $\mathcal{T}$ if and only if $L$ is a square lattice.

Proof. Suppose $H$ acts simply transitively on the tiles in $\mathcal{T}$. Since $L$ acts transitively on the tiles in $\mathcal{T}^{\prime}$ we have $H=L \cup L \rho \cup L \rho^{2} \cup L \rho^{3}$, so $L$ is the translation subgroup of $H$. We conclude by Lemma 2.2 that $L$ is a square lattice.

Conversely, suppose that $L$ is a square lattice. Then for every $\tau=x^{i} y^{j}$ in $L$ the $90^{\circ}$ rotation $x^{-j} y^{i}=\rho \tau \rho^{-1}$ of $\tau$ must also be in $L$. Therefore $\rho$ normalizes $L$, and hence, since $\rho$ has order 4, we get $H=L \cup L \rho \cup L \rho^{2} \cup L \rho^{3}$. Since $L$ acts simply transitively on the tiles in $\mathcal{T}^{\prime}$, this implies that $H$ acts simply transitively on the tiles in $\mathcal{T}$.

Proposition 3.3. Let $\sigma \in \Gamma$ be a glide reflection such that $P$ and $\sigma(P)$ do not overlap, and set $P^{\prime}=P \cup \sigma(P)$. Let $\mathcal{T}^{\prime}$ be a $P^{\prime}$-tiling of the plane by translations by a lattice $L \subset \mathbb{Z}^{2}$, and let $\mathcal{T}$ denote the $P$-tiling induced by $\mathcal{T}^{\prime}$. Then $H=\langle L, \sigma\rangle$ acts simply transitively on the tiles in $\mathcal{T}$ if and only if $\sigma^{2} \in L$ and $L$ is either a rectangular lattice with one side parallel to the axis of reflection, or contains such a lattice with index 2.

Proof. Suppose $H$ acts simply transitively on the tiles in $\mathcal{T}$. Since $L$ acts transitively on the tiles in $\mathcal{T}^{\prime}$ we have $H=L \cup L \sigma$, so $L$ is the translation subgroup of $H$. It follows immediately that $\sigma^{2} \in L$, and by Lemma 3.4 below we see that $L$ is a lattice of the required type.

Conversely suppose that $\sigma^{2} \in L$ and $L$ contains a lattice of the required type. We assume that the axis of $\sigma$ is vertical, since the other cases are almost identical. Then $L$ contains a lattice $\left\langle x^{a}, y^{b}\right\rangle$ with index at most 2, so for every $\tau=x^{i} y^{j}$ in $L$ the projection $x^{2 i}$ of $\tau^{2}$ onto the $x$-axis is also in $L$. It follows that $x^{-2 i} \tau=x^{-i} y^{j}=\sigma \tau \sigma^{-1}$ is in $L$. Therefore $\sigma$ normalizes $L$, and hence since $\sigma^{2} \in L$ we get $H=L \cup L \sigma$. Since $L$ acts simply transitively on the tiles in $\mathcal{T}^{\prime}$, this implies that $H$ acts simply transitively on the tiles in $\mathcal{T}$.

Lemma 3.4. Let $G$ be an infinite subgroup of $\Gamma$ which contains a glide reflection $\sigma$. Then the translation subgroup $L$ of $G$ is either a rectangular lattice with one side parallel to the axis of $\sigma$, or contains such a lattice with index 2.

Proof. We assume that the axis of $\sigma$ is vertical; the other three cases are similar. Let $\tau \in L$; then we can write $\sigma=x^{k} y^{l} r f$ and $\tau=x^{i} y^{j}$ for some $i, j, k, l \in \mathbb{Z}$. Then $\sigma \tau \sigma^{-1}=x^{-i} y^{j}$ is in $L$, and hence $\left(\sigma \tau \sigma^{-1}\right) \tau=y^{2 j}$ and $\left(\sigma \tau \sigma^{-1}\right)^{-1} \tau=x^{2 i}$ must also be in $L$. Therefore $L$ contains a rectangular lattice $\left\langle x^{a}, y^{b}\right\rangle$ with index at most 2.

Proposition 3.5. Let $\rho \in \Gamma$ be a $180^{\circ}$ rotation and let $\sigma \in \Gamma$ be a glide reflection. Assume that $P, \rho(P), \sigma(P)$, and $\rho \sigma(P)$ do not overlap, and set $P^{\prime}=P \cup \rho(P) \cup$ $\sigma(P) \cup \rho \sigma(P)$. Let $\mathcal{T}^{\prime}$ be a $P^{\prime}$-tiling of the plane by translations by a lattice $L \subset \mathbb{Z}^{2}$, and let $\mathcal{T}$ denote the $P$-tiling induced by $\mathcal{T}^{\prime}$. Then $H=\langle L, \rho, \sigma\rangle$ acts simply transitively 
on the tiles in $\mathcal{T}$ if and only if $\sigma^{2} \in L,(\rho \sigma)^{2} \in L$, and $L$ is either a rectangular lattice with one side parallel to the axis of reflection, or contains such a lattice with index 2.

Proof. Suppose $H$ acts simply transitively on the tiles in $\mathcal{T}$. Since $L$ acts transitively on the tiles in $\mathcal{T}^{\prime}$ we get $H=L \cup L \rho \cup L \sigma \cup L \sigma \rho$ and $H / L \cong Z_{2} \times Z_{2}$. It follows that $\sigma^{2} \in L,(\rho \sigma)^{2} \in L$, and $L$ is the translation subgroup of $H$. By Lemma 3.4 this last statement implies that $L$ is a lattice of the required type.

Conversely suppose that $\sigma^{2} \in L,(\rho \sigma)^{2} \in L$, and $L$ is a lattice of the required type. In the proof of Proposition 3.1 we showed that for a $180^{\circ}$ rotation $\rho$ we have $\langle L, \rho\rangle=L \cup L \rho$ and $\rho$ normalizes $L$. In the proof of Proposition 3.3 we showed that the assumption about the shape of $L$ implies that $\sigma$ normalizes $L$. Therefore $\sigma \rho \sigma^{-1}=$ $\sigma(\rho \sigma)^{2} \sigma^{-1} \rho^{-2}\left(\rho \sigma^{-2} \rho^{-1}\right) \rho$ is in $L \rho$, since $(\rho \sigma)^{2}, \rho^{-2}=1$, and $\sigma^{-2}$ are all in $L$, and $\sigma$ and $\rho$ normalize $L$. It follows that $\sigma$ normalizes $\langle L, \rho\rangle$. Since $\sigma^{2} \in L$ this implies $H=L \cup L \rho \cup L \sigma \cup L \rho \sigma$. Since $L$ acts simply transitively on the tiles in $\mathcal{T}^{\prime}$, this implies that $H$ acts simply transitively on the tiles in $\mathcal{T}$.

Proposition 3.6. Let $\rho \in \Gamma$ be a $90^{\circ}$ rotation and let $\sigma \in \Gamma$ be a glide reflection. Assume that $P, \rho(P), \rho^{2}(P), \rho^{3}(P), \sigma(P), \rho \sigma(P), \rho^{2} \sigma(P)$, and $\rho^{3} \sigma(P)$ do not overlap, and set

$$
P^{\prime}=P \cup \rho(P) \cup \rho^{2}(P) \cup \rho^{3}(P) \cup \sigma(P) \cup \rho \sigma(P) \cup \rho^{2} \sigma(P) \cup \rho^{3} \sigma(P) .
$$

Let $\mathcal{T}^{\prime}$ be a $P^{\prime}$-tiling of the plane by translations by a lattice $L \subset \mathbb{Z}^{2}$, and let $\mathcal{T}$ denote the $P$-tiling induced by $\mathcal{T}^{\prime}$. Then $H=\langle L, \rho, \sigma\rangle$ acts simply transitively on the tiles in $\mathcal{T}$ if and only if $\sigma^{2} \in L,(\rho \sigma)^{2} \in L$, and $L$ is a square lattice whose sides are either parallel to the coordinate axes or have slope \pm 1 .

Proof. Suppose $H$ acts simply transitively on the tiles in $\mathcal{T}$. Since $L$ acts transitively on the tiles in $\mathcal{T}^{\prime}$ we get

$$
H=L \cup L \rho \cup L \rho^{2} \cup L \rho^{3} \cup L \sigma \cup L \rho \sigma \cup L \rho^{2} \sigma \cup L \rho^{3} \sigma
$$

and $H / L \cong D_{8}$. It follows that $\sigma^{2} \in L,(\rho \sigma)^{2} \in L$, and $L$ is the translation subgroup of $H$. Lemma 2.2 implies then that $L$ is a square lattice, and Lemma 3.4 implies that the sides of the squares in $L$ are either parallel to the coordinate axes or have slope \pm 1 .

Conversely suppose that $\sigma^{2} \in L,(\rho \sigma)^{2} \in L$, and $L$ is a lattice of the required type. In the proof of Proposition 3.2 we showed that for a $90^{\circ}$ rotation $\rho$ and a square lattice $L$ we have $\langle L, \rho\rangle=L \cup L \rho \cup L \rho^{2} \cup L \rho^{3}$, and $\rho$ normalizes $L$. In the proof of Proposition 3.3 we showed that our assumptions about $L$ imply that $\sigma$ normalizes $L$. In addition, $\sigma \rho \sigma^{-1}=\left(\rho^{-1}(\rho \sigma)^{2} \sigma^{-2} \rho\right) \rho^{-1}$ is in $L \rho^{-1}$, since $(\rho \sigma)^{2}$ and $\sigma^{-2}$ are in $L$ and $\rho$ normalizes $L$. Therefore $\sigma$ normalizes $\langle L, \rho\rangle$. Since $\sigma^{2} \in L$ this implies

$$
H=L \cup L \rho \cup L \rho^{2} \cup L \rho^{3} \cup L \sigma \cup L \rho \sigma \cup L \rho^{2} \sigma \cup L \rho^{3} \sigma .
$$

Since $L$ acts simply transitively on the tiles in $\mathcal{T}^{\prime}$, we conclude that $H$ acts simply transitively on the tiles in $\mathcal{T}$. 
The final result in this section shows that we can avoid tilings which involve both glide reflections and $90^{\circ}$ rotations.

Proposition 3.7. Let $\mathcal{T}$ be an isohedral $P$-tiling of the plane and let $H \leq \Gamma$ be a group that acts simply transitively on the tiles in $\mathcal{T}$. Suppose that $H / L \cong D_{8}$, where $L$ is the translation subgroup of $H$. Then there is another isohedral $P$-tiling $\mathcal{T}^{\prime}$ and a group $H^{\prime} \leq \Gamma$ that acts simply transitively on the tiles in $\mathcal{T}^{\prime}$ such that $H^{\prime} / L^{\prime} \cong Z_{4}$, where $L^{\prime}$ is the translation subgroup of $H^{\prime}$.

Proof. Since $H / L \cong D_{8}$ the group $H$ contains $90^{\circ}$ rotations and glide reflections through axes in all four allowable directions. Therefore by Proposition $3.6 \mathrm{~L}$ is a square lattice whose sides are either parallel to the coordinate axes or have slope \pm 1 .

Suppose we are in the first case, where the square lattice $L$ has sides parallel to the coordinate axes. Then $L=\left\langle x^{a}, y^{a}\right\rangle$ for some $a \geq 1$. Let $\sigma=x^{k} y^{l} f \in H$ be a glide reflection through an axis of slope 1 such that $k+l$ has minimum absolute value. If $|k+l|>a / 2$ we could reduce $|k+l|$ by replacing $\sigma$ with $\left(x^{a}\right)^{t} \sigma$ for some integer $t$, so we must have $|k+l| \leq a / 2$. Since $\sigma^{2}=(x y)^{k+l} \in L$ we have $a \mid k+l$ and hence $k+l=0$. It follows that $H$ contains a pure reflection $\sigma$ through an axis of slope 1 . Since $H$ acts simply transitively on the tiles in $\mathcal{T}$ no tile can cross an axis of reflection in $H$. This is a contradiction because the tiles in $\mathcal{T}$ are polyominoes with sides parallel to the coordinate axes.

Now suppose we are in the second case, where the square lattice has sides of slope \pm 1 . Then $L=\left\langle x^{a} y^{a}, x^{a} y^{-a}\right\rangle$ for some $a \geq 1$. An argument similar to that used in the preceding paragraph shows that $H$ contains a pure reflection $\sigma$ through a vertical axis. Such a reflection may be written in the form $\sigma=x^{k} r f$. Since $x^{2 a i} \in L$ for all $i \in \mathbb{Z}$ we see that each $x^{2 a i} \sigma=x^{2 a i+k} r f \in H$ is a pure reflection through a vertical axis. Therefore $H$ contains pure reflections through vertical axes spaced evenly at distance $a$. Similarly we see that $H$ contains pure reflections through horizontal axes spaced evenly at distance $a$. These axes divide the plane into $a \times a$ squares. Since no tile can cross the axis of a pure reflection, each tile in $\mathcal{T}$ is contained in one of these $a \times a$ squares. Let $\rho \in H$ be a $90^{\circ}$ rotation with center $(p, q)$. If $(p, q)$ lies on the axis of some reflection $\sigma_{0}$, then $\sigma_{0} \rho$ is a pure reflection through an axis of slope \pm 1 , and we get a contradiction as in the preceding paragraph. Therefore $(p, q)$ must lie in the interior of one of the $a \times a$ squares.

Let $n$ be the area of $P$. Since the area of a fundamental region for $L$ is $2 a^{2}$ and $(H: L)=8$, we have $8 n=2 a^{2}$. It follows that an $a \times a$ square is tiled by four copies of $P$ rotated about $(p, q)$. An $a \times a$ square tiles the plane by translations by the lattice $L^{\prime}=\left\langle x^{a}, y^{a}\right\rangle$. This tiling by squares induces a $P$-tiling $\mathcal{T}^{\prime}$, and it follows from Proposition 3.2 that $H^{\prime}=\left\langle x^{a}, y^{a}, \rho\right\rangle$ acts simply transitively on the tiles in $\mathcal{T}^{\prime}$. By construction we clearly have $H^{\prime} / L^{\prime} \cong Z_{4}$.

\section{Algorithms}

In this section we give an algorithm which determines whether a polyomino $P$ admits an isohedral tiling, and provides such a tiling if one exists. According to Proposition 2.1, 
if $P$ admits an isohedral tiling $\mathcal{T}$, then there is a subgroup $H$ of the symmetry group $G(\mathcal{T})$ which acts simply transitively on the tiles in $\mathcal{T}$. Let $L$ be the translation subgroup of $H$; then since $H / L$ is isomorphic to a subgroup of $D_{8}$, the index $(H: L)$ is equal to $1,2,4$, or 8 . If $(H: L)=1$, then $\mathcal{T}$ is a lattice tiling. To begin we give a polynomial time algorithm that finds a lattice tiling if one exists.

The key is to find all subgroups $L$ of index $n$ in $\mathbb{Z}^{2}$. Suppose $\left(\mathbb{Z}^{2}: L\right)=n$, and let $\{(a, b),(c, d)\}$ be a generating set for $L$. Then the area of a fundamental region for $L$ is $n$, so the determinant of the matrix

$$
A=\left(\begin{array}{ll}
a & c \\
b & d
\end{array}\right)
$$

is equal to $\pm n$. By interchanging the two generators of $L$ if necessary we may assume that $\operatorname{det}(A)=n$. Conversely, given $A \in \mathbb{M}_{2}(\mathbb{Z})$ such that $\operatorname{det}(A)=n$, the columns of $A$ generate a subgroup $L$ of $\mathbb{Z}^{2}$ such that $\left(\mathbb{Z}^{2}: L\right)=n$.

Lemma 4.1. Let $A, B \in \mathbb{M}_{2}(\mathbb{Z})$ satisfy $\operatorname{det}(A)=\operatorname{det}(B)=n \geq 1$ and $A \equiv$ $B(\bmod n)$. Then the columns of $A$ and the columns of $B$ generate the same subgroup $L \leq \mathbb{Z}^{2}$. Moreover, the subgroup L satisfies $\left(\mathbb{Z}^{2}: L\right)=n$.

Proof. Let $L_{A}$ and $L_{B}$ be the subgroups of $\mathbb{Z}^{2}$ generated by the columns of $A$ and $B$. We observed above that $\left(\mathbb{Z}^{2}: L_{A}\right)=\left(\mathbb{Z}^{2}: L_{B}\right)=n$, so $n \mathbb{Z}^{2}$ is contained in both $L_{A}$ and $L_{B}$. Since $A \equiv B(\bmod n)$ we have $L_{A} / n \mathbb{Z}^{2}=L_{B} / n \mathbb{Z}^{2}$, and hence $L_{A}=L_{B}$.

To find generating sets for all subgroups $L \leq \mathbb{Z}^{2}$ of index $n$, it is sufficient to list all matrices $A \in \mathbb{M}_{2}(\mathbb{Z})$ with $\operatorname{det}(A)=n$, up to congruence modulo $n$. It is easy to obtain such a list as follows. For each congruence class in $\mathbb{Z}^{2} / n \mathbb{Z}^{2}$ choose a representative $(a, b) \in \mathbb{Z}^{2}$ such that $\operatorname{gcd}(a, b) \mid n$. Use the Euclidean algorithm to find all pairs of integers $(c, d)$ such that $a d-b c=n$, and choose representatives for each congruence class modulo $n$ of such pairs $(c, d)$. Denote by $\mathcal{A}(n)$ the finite set of matrices

$$
A=\left(\begin{array}{ll}
a & c \\
b & d
\end{array}\right)
$$

obtained in this manner.

For an $n$-omino $P$, let $C(P) \subset \mathbb{Z}^{2}$ denote the set of centers of the unit squares which constitute $P$. Set $C(P)-C(P)=\{\mathbf{x}-\mathbf{y} \mid \mathbf{x}, \mathbf{y} \in C(P)\}$, and define a matrix $M$ with integer entries by letting each vector in $C(P)-C(P)$ be a column of $M$.

\section{Algorithm $L$}

Input: $n$-omino $P$.

Output: If $P$ admits a lattice tiling, then two vectors are provided that generate the lattice. Otherwise the output is the message "No lattice tiling." 
Compute the set $\mathcal{A}(n)$ of matrices.

For each $A \in \mathcal{A}(n)$,

If no column of $A^{-1} M$ has two integer entries,

Return the columns $A_{1}, A_{2}$ of $A$;

Polyomino $P$ admits a tiling by the lattice generated by $A_{1}, A_{2}$. End

End

$P$ admits no lattice tiling.

Proof of the validity of Algorithm $L$. By Lemma 4.1 the subgroups $L \leq \mathbb{Z}^{2}$ of index $n$ are precisely those subgroups of $\mathbb{Z}^{2}$ generated by the columns of some matrix $A \in \mathcal{A}(n)$. If $\left(\mathbb{Z}^{2}: L\right)=n=\operatorname{area}(P)$, then $P$ tiles by translations by $L$ if and only if the coordinates of the unit squares of $P$ represent distinct cosets of $L$ in $\mathbb{Z}^{2}$. If $L$ is generated by the columns of $A$ this is equivalent to $A \mathbf{x}+\mathbf{p} \neq A \mathbf{y}+\mathbf{q}$ for all $\mathbf{p}, \mathbf{q} \in P$ and $\mathbf{x}, \mathbf{y} \in \mathbb{Z}^{2}$ with $\mathbf{x} \neq \mathbf{y}$. By the definition of $M$ this is equivalent to saying no column of $A^{-1} M$ has both entries in $\mathbb{Z}$.

We now describe a polynomial time algorithm which determines whether an $n$-omino $P$ admits an isohedral tiling. The algorithm is divided into five cases that correspond to the five geometric equivalence classes of proper subgroups of $D_{8}$. Note that by Proposition 3.7 we do not need to consider the case $H / L \cong D_{8}$. In each case the basic steps in the algorithm are the same.

\section{Algorithm $I$}

Input: $n$-omino $P$.

Output: If $P$ admits an isohedral tiling, then a set $S$ consisting or one, two, or four nonoverlapping copies of $P$ is produced, together with two vectors that generate a lattice $L$. The isohedral $P$-tiling is obtained by translating the tiles in $S$ by the lattice $L$. Otherwise the output is the message "No isohedral tiling."

For each case $k=1$ to 5 successively,

Compute the collection $\mathcal{P}_{k}$ of sets $S$ of nonoverlapping copies of $P$.

Compute the set $\mathcal{A}_{k}$ of matrices as described in the paragraph below.

For each set $S \in \mathcal{P}_{k}$,

Let $P^{\prime}=\bigcup_{Q \in S} Q$.

Apply Algorithm $L$ to $P^{\prime}$ with the set $\mathcal{A}_{k}$ of matrices.

If Algorithm $L$ returns matrix $A$,

Return $S$ and the two column vectors of $A$.

End

End

End

$P$ admits no isohedral tiling.

To complete the description of the above algorithm, the collection $\mathcal{P}_{k}$ of tile sets $S$ and the collection $\mathcal{A}_{k}$ of $2 \times 2$ matrices $A$ must be constructed for each $k=1, \ldots, 5$. In 
what follows, distinct tiles are always assumed to be nonoverlapping. Also, in each case, the tile $P^{\prime}=\bigcup_{Q \in S} Q$ is taken to be rookwise connected; this severely limits the number of elements in $\mathcal{P}_{k}$. Note, in fact, that in each of the five cases below, the computation of $\mathcal{P}_{k}$ and $\mathcal{A}_{k}$ is a polynomial time procedure.

Case 1: $S \in \mathcal{P}_{1}$ if $S=\{P\}$. This is the lattice case already described in Algorithm $L$. The set $\mathcal{A}_{1}$ is precisely the set $\mathcal{A}(n)$ of matrices computed there.

Case 2: $S \in \mathcal{P}_{2}$ if $S=\{P, \rho(P)\}$, where $\rho$ is a $180^{\circ}$ rotation. The rotation $\rho$ is taken to be about a point on the boundary of $P$. The set $\mathcal{A}_{2}$ is exactly the set $\mathcal{A}(2 n)$ of matrices.

Case 3: $S \in \mathcal{P}_{3}$ if $S=\left\{P, \rho(P), \rho^{2}(P), \rho^{3}(P)\right\}$, where $\rho$ is a $90^{\circ}$ counterclockwise rotation. The rotation $\rho$ is taken to be about a point on the boundary of $P$. The set $\mathcal{A}_{3}$ consists of congruence classes $(\bmod 4 n)$ of matrices of the form

$$
A=\left(\begin{array}{rr}
a & -b \\
b & a
\end{array}\right), \quad \text { where } \quad \operatorname{det}(A)=a^{2}+b^{2}=4 n .
$$

Case 4: $S \in \mathcal{P}_{4}$ if $S=\{P, \sigma(P)\}$, where $\sigma$ is a glide reflection. The set $\mathcal{A}_{4}$ consists of congruence classes $(\bmod 2 n)$ of matrices $A$ with $\operatorname{det}(A)=2 n$ which have the forms

$$
A=\left(\begin{array}{ll}
a & 0 \\
0 & b
\end{array}\right) \quad \text { and } \quad A=\left(\begin{array}{ll}
a & a / 2 \\
0 & b / 2
\end{array}\right)
$$

if the axis of $\sigma$ is parallel to a coordinate axis, or the forms

$$
A=\left(\begin{array}{rr}
a & -b \\
a & b
\end{array}\right) \quad \text { and } \quad A=\left(\begin{array}{ll}
a & (a-b) / 2 \\
a & (a+b) / 2
\end{array}\right)
$$

if the axis of $\sigma$ has slope \pm 1 . In addition, if we assume (without loss of generality) that the axis of the glide reflection $\sigma$ has slope 0 or 1 , the translation vector of the glide may be written as $(g, 0)$ or $(g, g)$. Then for the matrix $A$ to be in $\mathcal{A}_{4}$ we must have $a \mid 2 g$.

Case 5: $S \in \mathcal{P}_{5}$ if $S=\{P, \rho(P), \sigma(P), \rho \sigma(P)\}$, where $\rho$ is a $180^{\circ}$ rotation and $\sigma$ is a glide reflection. The set $P \cup \sigma(P)$ is taken to be rookwise connected, and the rotation $\rho$ can be taken to be about a point on the boundary of $P \cup \sigma(P)$. The set $\mathcal{A}_{5}$ consists of matrices with the same forms as in Case 4 with $2 n$ replaced by $4 n$. In addition, if we assume (without loss of generality) that the axis of the glide reflection $\sigma$ has slope 0 or 1 , then the translation vector of the glide $\sigma$ may be written as $(g, 0)$ or $(g, g)$, and the translation vector of the glide $\rho \sigma$ may be written as $\left(0, g^{\prime}\right)$ or $\left(-g^{\prime}, g^{\prime}\right)$. Then for the matrix $A$ to be in $\mathcal{A}_{5}$ we must have $a \mid 2 g$ and $b \mid 2 g^{\prime}$.

Theorem 4.2. For any polyomino $P$ it can be decided in polynomial time whether there exists an isohedral P-tiling of the plane.

Proof. We will show that Algorithm I correctly decides. It is sufficient to prove that (1) if an isohedral $P$-tiling exists, then the algorithm produces a $P$-tiling, and (2) if the algorithm produces a $P$-tiling, then it is indeed isohedral. 
Assume the existence of an isohedral $P$-tiling $\mathcal{T}$. According to Proposition 2.1 there is a subgroup $H$ that acts simply transitively on the tiles in $\mathcal{T}$. Let $L$ be the translation subgroup of $H$. Then $H / L$ is isomorphic to a subgroup of $D_{8}$, and we may assume this subgroup is proper by Proposition 3.7. For any copy $P_{0}$ of $P$ in $\mathcal{T}$, the set $P^{\prime}=$ $h_{1}\left(P_{0}\right) \cup \cdots \cup h_{k}\left(P_{0}\right)$ is a fundamental region for the action of $L$ on the plane if and only if $\left\{h_{1}, \ldots, h_{k}\right\}$ is a set of coset representatives of $H / L$. If $\mathcal{T}^{\prime}$ is the tiling of the plane by translations of $P^{\prime}$ by the lattice $L$, then the tiling $\mathcal{T}$ is exactly the $P$-tiling induced by $\mathcal{T}^{\prime}$. The possibilities for coset representatives $h_{1}, \ldots, h_{k}$ can be determined by considering the geometric equivalence classes of proper subgroups of $D_{8}$. The following list is exhaustive.

1. $H=L$.

2. $H=\langle L, \rho\rangle$, where $\rho \in \Gamma$ is a $180^{\circ}$ rotation. The coset representatives are $\{1, \rho\}$.

3. $H=\langle L, \rho\rangle$, where $\rho \in \Gamma$ is a $90^{\circ}$ rotation. The coset representatives are $\left\{1, \rho, \rho^{2}, \rho^{3}\right\}$.

4. $H=\langle L, \sigma\rangle$, where $\sigma \in \Gamma$ is a glide reflection. The coset representatives are $\{1, \sigma\}$.

5. $H=\langle L, \rho, \sigma\rangle$, where $\rho \in \Gamma$ is a $180^{\circ}$ rotation and $\sigma \in \Gamma$ is a glide reflection. The coset representatives are $\{1, \rho, \sigma, \rho \sigma\}$.

There exist coset representatives $h_{1}, \ldots, h_{k}$ for $H / L$ such that the fundamental region $P^{\prime}=h_{1}\left(P_{0}\right) \cup \cdots \cup h_{k}\left(P_{0}\right)$ is rookwise connected. To see this, consider Case 5; the argument in the other cases is similar but easier. Since the subgroup $\langle L, \rho\rangle=L \cup L \rho$ has index 2 in $H$, there are two orbits for the action of $\langle L, \rho\rangle$ on the tiles in $\mathcal{T}$. Clearly there must exist two adjacent tiles $P_{1}$ and $P_{2}$ in different orbits. Since $H$ acts transitively on the tiles, there is an element $\sigma^{\prime} \in H$, necessarily a glide reflection, such that $P_{2}=\sigma^{\prime}\left(P_{1}\right)$. Let $Q=P_{1} \cup P_{2}$ denote the rookwise connected union of these two tiles. Since

$$
H=\left\langle L, \rho, \sigma^{\prime}\right\rangle=\langle L, \rho\rangle \cup\langle L, \rho\rangle \sigma^{\prime}
$$

we see that the orbit of $Q$ under $\langle L, \rho\rangle$ is a tiling $\mathcal{T}^{\prime}$ of the plane. There are two orbits of tiles in $\mathcal{T}^{\prime}$ under the action of $L$, and as above there must be adjacent tiles $Q_{1}$ and $Q_{2}$ in different orbits. Then $Q_{1} \cup Q_{2}$ is a rookwise connected fundamental region for $H$ made up of tiles in $\mathcal{T}$.

We have shown that the set of possibilities for the tile $P^{\prime}$ in tiling $\mathcal{T}^{\prime}$ is exactly the sets called $\mathcal{P}_{k}, k=1, \ldots, 5$, enumerated in Algorithm I. For Cases 3-5, the propositions in Section 3 place restrictions on a corresponding lattice $L$ if an isohedral tiling is to exist. These restrictions correspond exactly to the restrictions placed on the matrices $A \in \mathcal{A}_{k}$ whose column vectors generate the translation lattice in Algorithm $I$. What Algorithm $I$ does is precisely this. It checks, for each of the five cases, every possible appropriate rookwise connected set $\left\{h_{1}(P), \ldots, h_{k}(P)\right\}$ and uses Algorithm $L$ to search for any viable corresponding translation lattice $L$. Therefore, if an isohedral $P$-tiling exists, Algorithm $I$ will produce a $P$-tiling. Conversely, if Algorithm $I$ does produce a $P$-tiling $\mathcal{T}$, then the propositions in Section 3 ensure that the group $H$ acts simply transitively on the tiles of $\mathcal{T}$ and, therefore, that $\mathcal{T}$ is indeed isohedral. 


\section{References}

1. D. Beauquier and M. Nivat, On translating one polyomino to tile the plane, Discrete Comput. Geom. 6 (1991), 575-592.

2. K. Dahlke, A heptomino of order 76, J. Combin. Theory Ser. A 51 (1989), 127-128; erratum in J. Combin. Theory Ser. A 52 (1989), 321.

3. S. W. Golomb, Polyominoes, Scribner, New York (1965).

4. S. W. Golomb, Polyominoes which tile rectangles, J. Combin. Theory Ser. A 51 (1989), 117-124.

5. S. W. Golomb, Polyominoes, second edition, Princeton University Press, Princeton, NJ (1994).

6. S. W. Golomb, Tiling rectangles with polyominoes, Math. Intelligencer 18 (1996), 38-47.

7. S. W. Golomb, Tiling with sets of polyominoes, J. Combin. Theory 9 (1970), 60-71.

8. B. Grünbaum and G. S. Shephard, Tilings and Patterns, Freeman, New York (1987).

9. D. A. Klarner, Packing a rectangle with congruent $n$-ominoes, J. Combin. Theory 7 (1969), 107-115.

10. W. R. Marshall, Packing rectangles with congruent polyominoes, J. Combin. Theory Ser. A 77 (1997), 181-192.

11. R. Penrose, Pentaplexity, Math. Intelligencer 2 (1979), 32-37.

12. I. M. Stewart and A. Wormstein, Polyominoes of order 3 do not exist, J. Combin. Theory Ser. A 61 (1992), $130-136$.

13. H. Wang, Games, logic, and computers, Scientific American 213 (1965), 98-106.

14. H. A. G. Wijshoff and J. van Leeuwen, Arbitrary versus periodic storage schemes and tessellations of the plane using one type of polyomino, Inform. and Control 62 (1984), 1-25.

Received June 23, 1997, and in revised form April 6, 1998. 